\title{
Implementation of Neutrosophic-Based Decision Support System for Effective Diagnosis of Liver Disease
}

\author{
Okpako, A.E ${ }^{1 *}$, Omoghenemuko, G.I. ${ }^{2}$, Odikwa, H.N ${ }^{3}$ \\ ${ }^{I}$ Department of Computer Science, Edwin Clark University, Kiagbodo Delta State, Nigeria \\ ${ }^{2}$ Department of Computer Science, College of Education, Warri Delta State, Nigeria \\ ${ }^{3}$ Department of Computer Science, Abia State, University, Uturu, Abia State, Nigeria \\ Corresponding author* \\ DOI: https://dx.doi.org/10.51244/IJRSI.2021.8403
}

\begin{abstract}
Liver diseases have been shown to be highly correlated to excessive consumption of alcohol and other harmful or injurious substances such as drugs and toxins. The Nigeria social milieu cannot do away with excessive consumption of alcoholicrelated substances and drugs, which are predominantly consumed on weekends either in parties or clubs. Most Nigeria teenagers and adults alike who are supposedly considered as socially correct indulge in excessive consumption of alcohol and other harmful substances leaving the alcoholic companies and shops smiling to the banks. This unpalatable trend has dire consequences as it raises the figure of liver disease patients, which is mostly confused with other tropical diseases like malaria and as such its manifestation cannot be predicted on time with certainty. Timely diagnosis is a panacea to the management of the disease but this is not the case most times as there are handful of hepatologists that can adequately diagnose this disease since General practitioner might not be able to diagnose them on time. This research seeks to comparatively analyze the performance of Neutrosophic-based Decision Support System and Multilayer Neural Network (Traditional Neural Network) in the classification of Indian Liver Patient Dataset (ILDP) as well as articulate its suitability in the classification or diagnosis of liver disease (ILPD). Object Oriented Analysis and Design methodology was used while the implementation was done using WEKA and Java on a Netbeans platform. Experimental results show that Neutrosophic-Based Decision Support System (NBDSS) with an accuracy of $96.41 \%$ using a confusability measurement threshold of 0.003278 performed better than the conventional neural network with an accuracy of $72.45 \%$. This clearly shows that Neutrosophic-based Decision Support System is suitable for the diagnosis of liver diseases.
\end{abstract}

Keywords: Neutrosophic, Liver disease, fuzzy logic

\section{INTRODUCTION}

$\mathrm{T}$ here has been exponential growth in the frontiers of decision support systems as applied to medical diagnosis in recent times which is traceable to the active participation and collaboration of computer science and medical researchers. Information technology viz-a-viz machine learning has broaden the reach and impact of medicine to people and is seemingly adding value more and more in terms of service delivery. Developing country like Nigeria needs information technology to expand both in length and breadth of its economy. Trends in the medical sector suggest that the future is more computer enabled, more connected and more reliant on computing devices and its related technologies which would ultimately have positive impact on both patients and medical personnel. Medical diagnosis would move more and more from hospitals into patient environment by computing devices.

The human body system like every other system is made up several components that work together in achieving the overall objective of a healthy human body system. Liver is one of the five organs of the human body that works in synergy with others to achieve the wellbeing of humans or animals whose function cannot be hurriedly emphasized. It is the biggest organ in the human body which can withstand stress to a superlative degree. It is majorly responsible for the metabolic activities of the human body; however, due to its special nature, it is prone to several injurious agents such as toxins and drugs. Hepatitis and Cirrhosis are the two major types of liver disorder. Hepatitis is caused by several factors such as virus, inherited disorders, alcohols and drugs, is the inflammation of the liver while Cirrhosis caused by excessive alcohol consumption, chronic infection, inherited disorder of iron and copper metabolism, severe reactions to certain medication and fatty liver is a condition where the liver responds to injury. The prevalence of liver disorders varies from region to region possibly due to the peculiarities of injurious agents in that region or other etiological factors associated with it. For example, in Enugu state in Nigeria, [12] opined that etiological factors identified were

hepatitis B virus (HBV), hepatitis C virus (HCV), alcohol, smoking and cryptogenic.

Several researchers have pointed out the negative impact of liver disease such as the work of [12], the work of [1] which reported that cirrhosis was the second only to hepatocellular carcinoma in causing liver disease. According to [1], liver cirrhosis and primary liver cancer account for two thirds of liver diseases in hospitalized patients in Nigerian tertiary hospitals. It is revealed from the work of [12] that the patients whose liver disease could not be classified are a very 
important group and should be treated with a firsthand treatment so as to timely diagnose such in future. The reasons for not classifying them include very late presentation with little or no time to conclude investigations before death and lack of adequate laboratory support to make the correct diagnosis. The reason stated above is in tandem with the problems of confusable diseases and thus necessitated this research work in finding a suitable decision support system to ameliorate the embarrassing situation.

There is an emerging reality in medical decision support towards the use of clinical decision support systems to assist medical practitioners in making near accurate diagnosis through the use of machine learning algorithms. The artificial neural networks and its hybrids have been copiously applied in this regard and its performances have been seemingly encouraging yet there is still room for improvement. Whereas Neutrosophic logic is a new and emerging logic well suited to handle indeterminate and incomplete information. This paper proposes a hybrid of neutrosophic logic and neural network with a view of presenting an innovative method towards a better diagnosis of liver that would reduce mortality rate of patients.

\section{LITERATURE REVIEW}

[6] presented and compared C4.5, multilayer perception classifier, random forest classifier, support vector machine classifier and Bayesian classifier for classification of liver patient data. They opined that random forest gave a highest accuracy of $71.86 \%$. [16] also proposed the use of multilayer perceptron for the classification of liver patient data which was compared to other classification techniques. They opined that multilayer perceptron with random gives highest accuracy of $74.7826 \%$ using BUPA liver disorder dataset while nearest neighbor with CFS gives highest accuracy of $73.07 \%$ for ILPD dataset. [4] have used Naïve Bayes and Forest Tree (FT) techniques to classify the liver patient data. Results show that Naïve Bayes gave a better performance with $75.54 \%$ of accuracy than Forest tree that has $72.66 \%$ of accuracy. [3] presented a classification of liver diseases using different classification technique. They opined that random forest algorithm performed better than other tree techniques with an accuracy of $79.22 \%$ using a 80-20 rule. [18] used Support Vector Machine (SVM) and Naïve Bayes algorithms for classification of liver patients where Indian Liver Dataset (ILPD) was used for performance evaluation. Their experimental result shows that Support Vector Machine (SVM) is better than Naïve Bayes algorithm in classifying liver patient dataset. [15] used a multilayer perceptron to classify liver patient dataset and had a classification accuracy of $77.77 \%$. [7] presented a computational intelligence technique for liver patient classification whose work indicated that Random Forest oversampling with $200 \%$ outperformed all the other techniques. [10] presented an algorithm for Hepatitis diagnosis using Principal Component Analysis (PCA) and Minimized Extreme Learning Machine (EMELM). The PCA was used for the feature phase which brought the number of features from 19 to 6 and its performance was comparatively better. However, about $48.7 \%$ of the data in the UCI ( University of California Irvine) datasets was not used as they contain missing information or data. [9] proposed a Generalized Regression Neural Network (GRNN) based expert system for the diagnosis of the Hepatitis B virus disease. The system classifies each patient into infected and non-infected as well as the degree of infection. [13] presented a decision support system for Hepatitis $\mathrm{B}$ and $\mathrm{C}$ using multilayer perceptron Feed Forward Neural Network and had an accuracy of $91.2 \%$.

[6] presented a hybrid classification system for $\mathrm{HCV}$ diagnosis using modified Particle Swarm Optimization Algorithm and Least Square Support Vector Machine. Principal component analysis was used to extract the feature vector. The system had a comparative better performance. [17] presented an automatic diagnosis system based on neural network for Hepatitis virus by combining feature extraction and classification and they made use of UCI datasets. [7] presented PCA-NB algorithm to improve the predictive accuracy of hepatitis classification using the hybrid of Principal component analysis for feature evaluator and ranking, and Naïve Bayes algorithm for classification. It was tested using UCI datasets. [11] presented a classification approach for Hepatitis disease diagnosis by combining support vector machine with scatter search approach called 3SVM. They made use of UCI datasets and had accuracy rate of $98.75 \%$. [5] implemented a medical prediction system for diagnosing Hepatitis making use of Artificial Neural Network. [2] proposed Neutrosophic classifier which employs Neutrosophic logic for its working and is an extension of commonly used fuzzy classifier. They compared it with the commonly used fuzzy classifiers on the following parameters: nature of membership functions, number of rules and indeterminacy in the results generated. They proved that extended fuzzy classifier: Neutrosophic classifier; optimize the said parameters when compared with the fuzzy counterpart. Finally, they concluded that though Neutrosophic logic is still in its nascent stage, but it has the potential to be experimented for further exploration in different domains.

One significant work towards use of Neutrosophic logic has been presented by [8], where Neutrosophic logic based extension of fuzzy classifier was designed. Although, fuzzy concept lattices have been worked upon by many researchers, this is the first attempt to further generalize concept lattices with Neutrosophic logic. [9] introduced an emerging approach for Intrusion Detection System using Neutrosophic Logic Classifier which is an extension/combination of the fuzzy logic, intuitionistic logic, paraconsistent logic, and the threevalued logics that use an indeterminate value. The proposed method partitions the dataset into normal, abnormal and indeterminate based on the degree of membership of truthfulness, degree of membership of indeterminacy and degree of membership of falsity. The Neutrosophic Logic Classifier generates the Neutrosophic rules to determine the intrusion in progress. Improvised genetic algorithm is adopted 
in order to detect the potential rules for performing better classification. Their work exhibits the efficiency of handling uncertainty in intrusion detection precisely using Neutrosophic Logic Classifier based Intrusion Detection System. [11] used Neutrosophic and fuzzy C means clustering for improving Computer Tomography (CT) liver image. The abdominal CT imaging has been chosen and segmentation approach has been applied to see their ability and accuracy to segment abdominal CT images. The abdominal CT image is transformed into Neutrosophic domain, which is described using three subsets. The percentage of truth in the subset $\mathrm{T}$, the percentage of indeterminacy in a subset $\mathrm{I}$, the percentage of falsity in a subset F. threshold for subset T, I, F is adapted using fuzzy C mean algorithm. He concluded that Neutrosophy can reduce over segmentation and gives a better performance on noisy and non-uniform images obtained by using other methods since the technique can handle uncertainty and indeterminacy better.

\section{THEORETICAL FRAMEWORK}

Central to the proposed algorithm is the machine learning algorithm called neural network, which is used for the classification task after the suitable number of features have been selected through the feature selection component; though in this system, feature selection was not used. The distributions of each of the instances are actually calculated in order to determine the reliability metric to measure the indeterminacy in the datasets. The varied pattern and values are analyzed and a suitable threshold is used to adequately classify the instances into their respective classes.

\subsection{Neural Network}

This is a black box supervised learning algorithms used widely in designing and programming several intelligent systems whose working principle is in tandem with natural biological neurons. Neural networks are based on simulated neurons that are joined together in a variety of ways to form networks. Neural network resembles the human brain in the following two ways: -

\section{a. A neural network acquires knowledge through learning \\ b. Neural network knowledge is stored within the interconnection strengths known as synaptic weight.}

Neural networks are typically organized in layers. Layers are made up of a number of interconnected nodes, which contain an activation function. Patterns are presented to the network via the input layer, which communicates to one or more hidden layers where the actual processing is done via a system of weighted connections.

\subsection{Neutrosophic Logic}

It is an open truth that scientists in the quest to unravel the underlying course and effect of any phenomenon have always look for typical and normal phenomenon but medical sciences is orthogonal in this regard to conventional sciences as it looks into atypical, abnormal and morbid phenomenon. It is the central purpose of medical science to finding a law or relation for these abnormal, atypical and sometimes morbid phenomena in order to characterize such situations. Ludwik Fleck (1896-1961), a polish philosopher and physician was the first to notice this and rightly presented it in his book titled "Genesis and Development of a scientific fact" in 1926. He opined that there are some specific features of the medical way of thinking. He also went further to state that there was no sharp border between these phenomena. Smooth transitions in the space of diseases feed from one phenomenon and very small variations could be the reasons why a physician diagnoses a patient with $\mathrm{x}$ instead of $\mathrm{y}$. It is an established conception that physician tends to rely on experience and intuition and not on rational and strong rules to deduce from the patients' data to ascertain a disease. Fuzzy logic proposed by Lofti Zadeh on the other hand, deals with the notion of degree of belongingness in which value ranges between 0 and 1 or true or false respectively. The degree is expressed as the membership function of a mapping from a set $\mathrm{A}$ to the interval $[0,1]$ using a linguistic variable. Membership function denoted by $A_{s}(x)$ represents a unique fuzzy set $\mathrm{S}$. This logic is quite different from previous logic theory because rather than considering the categorical boundaries of 1 and 0 only for classes, it seeks for the degree of membership into the respective classes or sets. If the value is 0 , it means that the element does not belong to the fuzzy set $\mathrm{S}$, otherwise 1 , which means that it belongs fully to the fuzzy set $\mathrm{S}$. This is best captured in function below.

$$
\mathrm{A}_{\mathrm{s}}(\mathrm{x}): \mathrm{X} \rightarrow[0,1]
$$

[19] stated that "In a given pathology, we denote S a set of symptoms, $\mathrm{D}$ a set of diagnoses and $\mathrm{P}$ a set of patients. What we call medical knowledge is a fuzzy relation, generally denoted by $\mathrm{R}$, from $\mathrm{S}$ to $\mathrm{D}$ expressing associations between symptoms or syndromes, and diagnoses or group of diagnoses".

The Neutrosophic Logic proposed by [17] is an extension of fuzzy logic which ensures that in addition to the membership to truth and falsity as presented in [19], it caters for issues of uncertainty, ambiguity, redundancy, imprecision, undefinedness, unknown, incompleteness and other varied meanings of indeterminacy. It is a logic in which each proposition is estimated to have the percentage of truth in a subset $T$, the percentage of indeterminacy in a subset $I$, and the percentage of falsity in a subset $F$, where $T$, I, F are defined above, is called Neutrosophic Logic.

A Neutrosophic set $A$ in $X$ is characterized by a truth membership function TA, an indeterminacy- membership function IA and a falsity-membership function $F A$. TA(x), $\mathrm{IA}(\mathrm{x})$ and $\mathrm{FA}(\mathrm{x})$ are real standard or non-standard subsets of ]$-0,1+[$. 


\subsection{Class Conditional Density Functions}

The probability distribution is defined as a function over general sets of values, or it may refer to the cumulative distribution function. Probability density functions have been used in statistics and probability theory for the density of continuous variables whose value at any given time in the set of possible values taken at random can provide a relative likelihood that the value of the random variables would equal that sample. To put it in a simpler form, while the absolute likelihood from a continuous variable to take any particular value is 0 , the value of the PDF at two different samples can be used to infer, in any particular situation or event of a random draw of the variable, how much likely it is that the random variable would equal one sample compared to the other sample. This PDF is used to specify the probability of any sample falling into any class of the dataset as opposed to only one class. The PDF over the entire dataset or sample space is equal to 1 .

\section{ARCHITECTURE}

The architecture of this system is adopted from the work of [14]. The framework as enshrined in their work was implemented using the Indian Liver Patient Dataset. The Neutrosophic logic enables our proposed system to have a reliability metric, which would act as a diagnostic criterion for data samples or points to be definitely diagnosed or definitely excluded in non-linear decision boundary cases. This metric quantifies any neutralities or inherent form of uncertainties or indeterminacy that can have negative impact in such classification. Thus the quantified indeterminacy is utilized to know the extent of quality in any predicted instance and ultimately improve the classification performance.

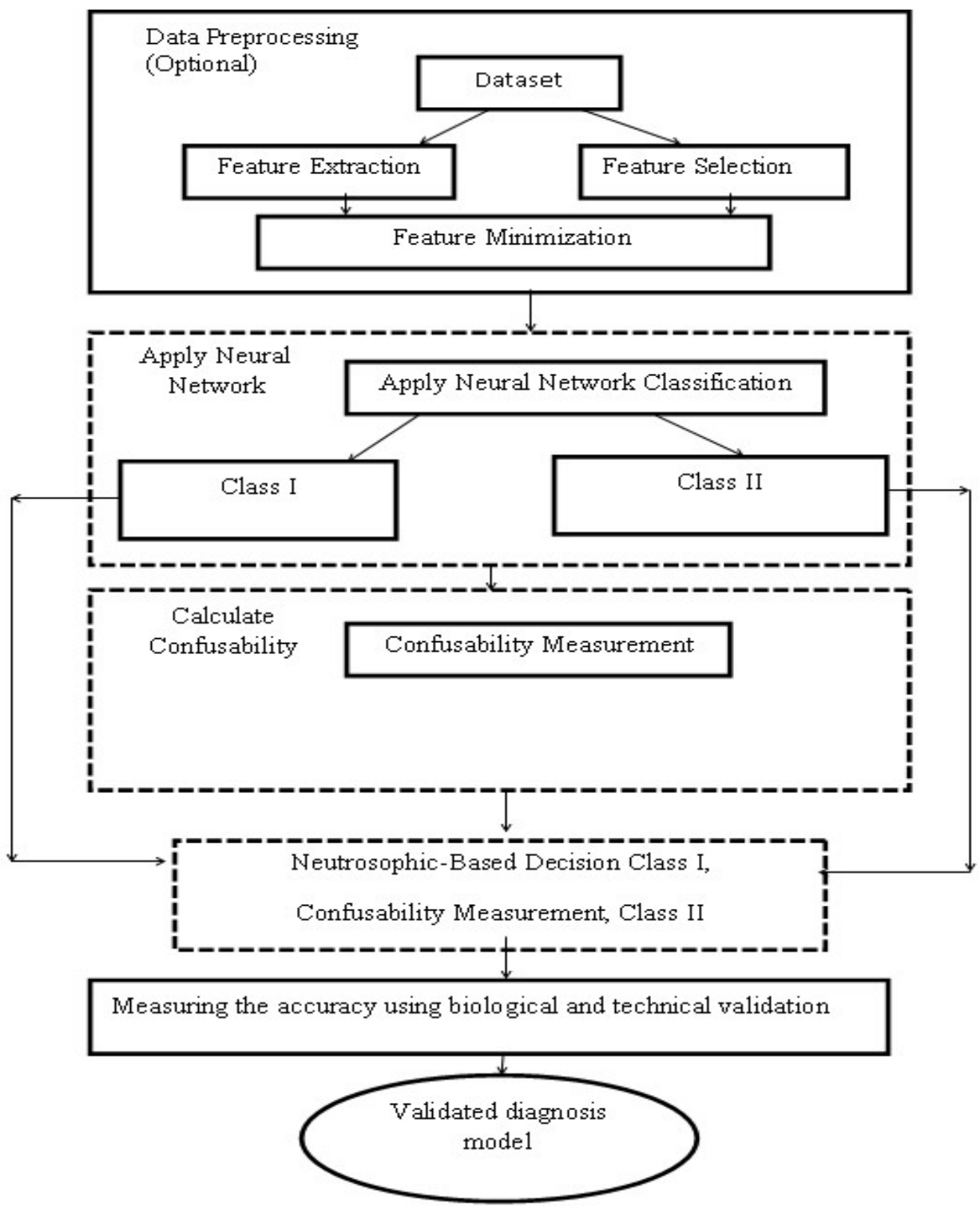

Fig. 1: Architecture of the proposed system (Source: [14]) 
Figure 1 depicts the architecture of the proposed system wherein the features are the values assigned to the datasets column-wise, which are then fed into the neural network. The neural network used in this research contain 23 (Twentythree) input neurons, three (3) hidden neurons, a learning rate of 0.3 , a momentum of 0.2 , an epoch of 1750 and 2 (two) output neurons after careful experimental analysis. In the training procedure, the methods mentioned in Figure 1 is used to train the neural network, calculates the parameter of the sigmoid functions and correction models and estimate the threshold values of confusability measurement from validation instances which is consequently used for the testing instances. The features of Hepatitis and Liver Disorder are combined to form the feature space of both training and testing procedure.

In the testing procedure, for each instance, the confusability measurement is obtained from which a suitable threshold for confusability measurement is determined and used for assigning the class labels accordingly.

\subsection{Machine Learning Software Implementation Tools}

The java technologies are fast becoming one of the suitable machine learning enabled programming languages due to its vast arrays of machine learning supporting libraries that can be used with it such as weka.jar and encog.jar, just to mention a few.

The implementation tools used are:

1. Netbeans 7.3 Integrated Development Environment (IDE)

2. WEKA.jar (Waikato Environment for Knowledge Analysis version 3.8.1)

3. jcommons.jar, jmathplot.jar,jama.jar,jfreechart.jar

With the aid of these libraries, machine-learning capability is infused into the java platform and as such one has a feel of the machine learning. A training set of 416 instances obtained from the UCI dataset of Indian Liver Patient Dataset was used and the system mean square error was recorded. Figure 2 is the signs and symptoms interface, which is used by the user to interact with system. The response of the system is a diagnostic recommendation from the system which would dictate the next line of action. A test data of 167 which represents $30 \%$ of the dataset is used to test and study the performance of the system by presenting the test pattern.

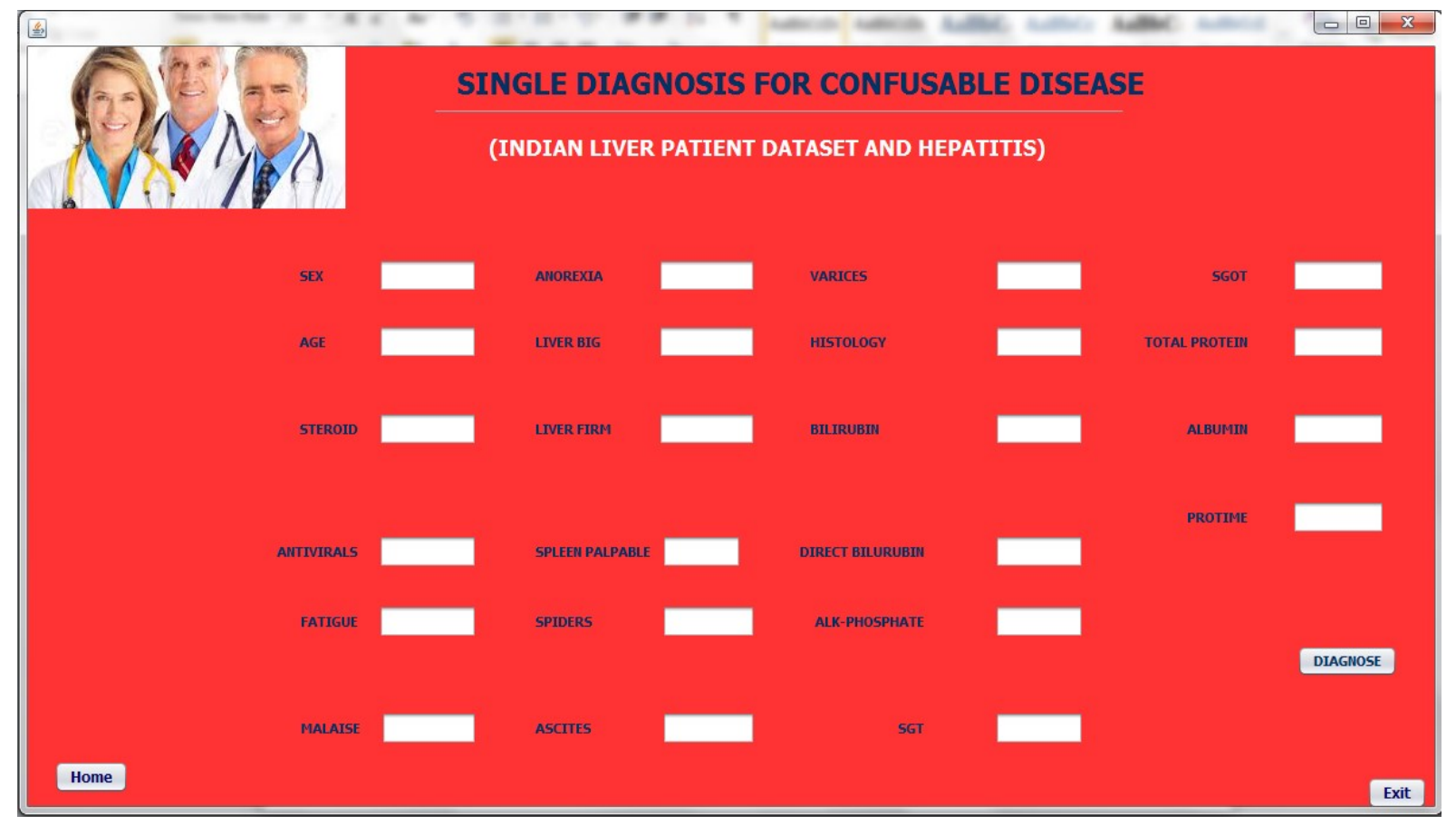

Fig.2: Features Interface

\section{EXPERIMENTAL RESULT OF INDIAN LIVER PATIENT DATASET (ILPD)}

Our proposed system was tested using ILPD in order to assay the performance of our model with the conventional neural network black box classification. This approach was to demonstrate the effectiveness of using the confusability measurement as part of the decision process in order to adequately diagnose or classify liver disease. The result of our proposed system (NBDSS) and the traditional MLP is shown in Table 1 while the graphical display of the result is shown with a bar chart in Figure 3 and 4 respectively.

Table 1: Performance Comparison using the Proposed Model and Traditional Approach (MLP) for Diagnosing Indian Liver Patient Dataset 


\begin{tabular}{|c|c|c|c|c|}
\hline & \multicolumn{2}{|c|}{$\begin{array}{c}\text { Traditional } \\
\text { Approach(MLP) }\end{array}$} & \multicolumn{2}{c|}{ Our Proposed Model } \\
\hline & Class 1 & Class 2 & Class 1 & Class 2 \\
\hline $\begin{array}{c}\text { Correctly } \\
\text { classified }\end{array}$ & 108 & 13 & 124 & 37 \\
\hline $\begin{array}{c}\text { Incorrectly } \\
\text { classified }\end{array}$ & 19 & 27 & 3 & 3 \\
\hline TP rate & 0.8500 & 0.3250 & 0.9764 & 0.9250 \\
\hline FP Rate & 0.6750 & 0.1500 & 0.0750 & 0.0236 \\
\hline Precision & 0.8000 & 0.4060 & 0.9674 & 0.9250 \\
\hline Recall & 0.8674 & 0.3251 & 0.9674 & 0.9250 \\
\hline F-Measure & 0.8240 & 0.3610 & 0.9674 & 0.9250 \\
\hline Accuracy & \multicolumn{2}{|c|}{$72.45 \%$} & \multicolumn{2}{c}{$96.41 \%$} \\
\hline
\end{tabular}

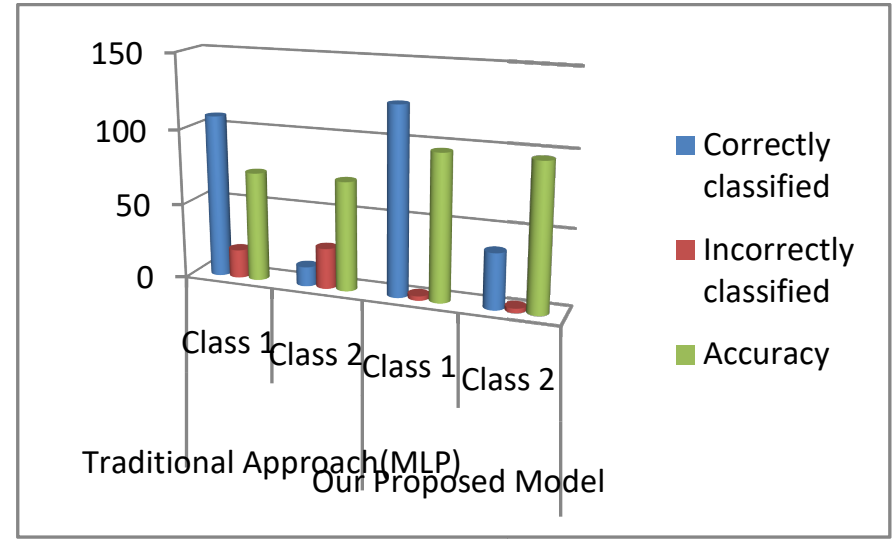

Fig. 4: Performance analysis of proposed system with traditional approach using ILDP dataset

\section{DISCUSSION OF INDIAN LIVER PATIENT DATASET RESULTS}

The Indian Liver Patient Dataset (ILPD) has two classes designated as 1 (Positive) and 2 (Negative) which are observed from the scattered plot shown in Figure 5 to be overlapping hence could be confusing in terms of prediction. This dataset has overlap problem. Our model was tested using this dataset.

A confusability measurement threshold of 0.003278 was estimated which was clearly used to assign the class labels accordingly. The prediction table for the ILPD is shown in Table 2. Confusability measurement is greater than 0.003278 for all positive instances while negative instances should be less than 0.003278 and if any of the prediction goes contrary, such prediction should be updated. The class label " 1 " is used to denote liver disease patient while " 0 " is used to denote nonliver patient. It could be seen that with our approach the accuracy of the model is increased from $72.45 \%$ to $96.41 \%$ as presented in the results which is about $24 \%$ increase.

Fig 3: Performance analysis of proposed system with traditional approach using ILDP dataset

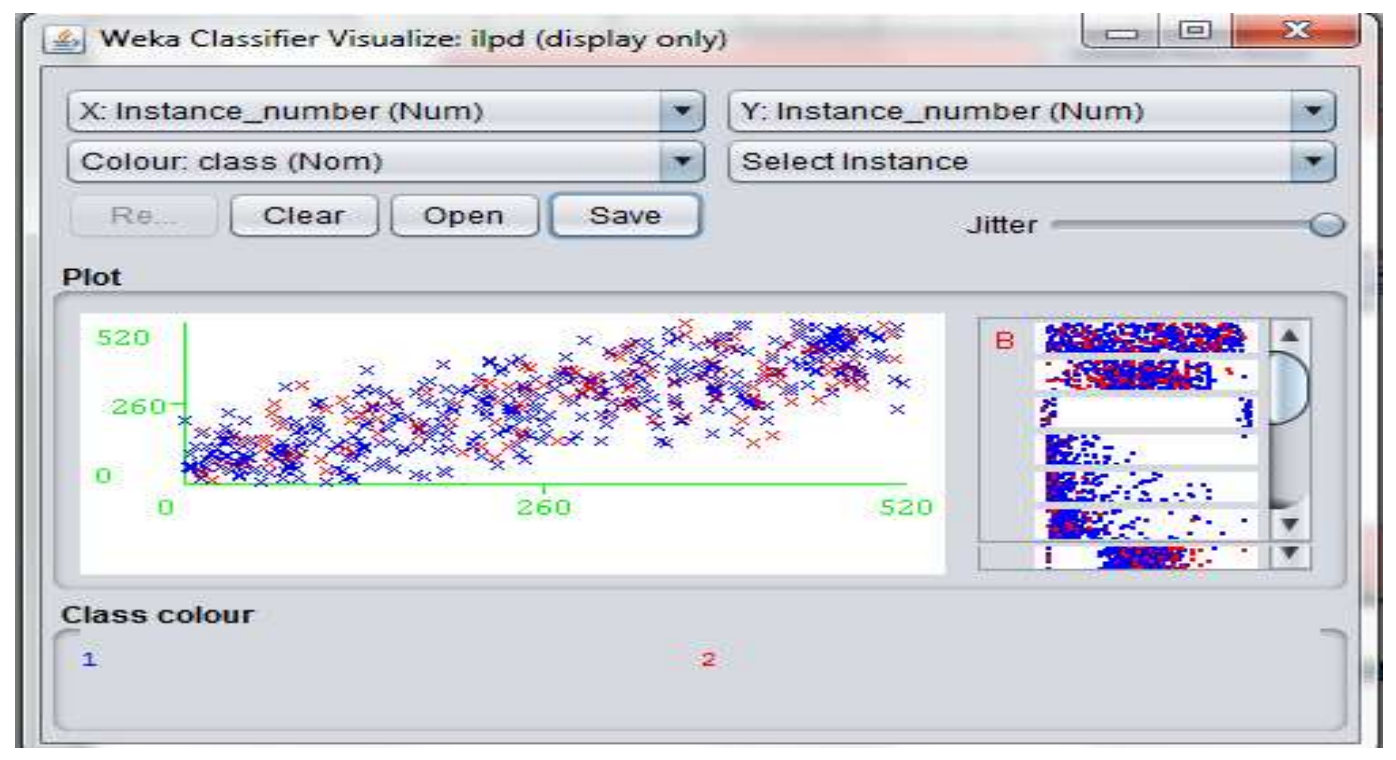

Fig. 5: Scattered plot of Indian Liver Patient Dataset 
Table 2: Prediction Table for ILPD Using our Proposed Model

\begin{tabular}{|c|c|c|c|c|c|}
\hline \multicolumn{4}{|c|}{ Prediction table using NBDSS(Our Model) } & \multicolumn{2}{|c|}{\begin{tabular}{|l|l|l|}
$\square$ & $\square$ & $\mathbf{x}$ \\
\end{tabular}} \\
\hline Index & Actual & Predicted & Error & confusability me... & \\
\hline 1 & 1.0 & 1.0 & & $0.00327675055 \ldots$ & $\Delta$ \\
\hline 2 & 1.0 & 1.0 & & $0.00353026942 \ldots$ & \\
\hline 3 & 1.0 & 1.0 & & $0.00476125574 \ldots$ & \\
\hline 4 & 1.0 & 1.0 & + & $0.00325121939 \ldots$ & \\
\hline 5 & 1.0 & 0.0 & & $0.00346752173 \ldots$ & \\
\hline 6 & 1.0 & 1.0 & & $0.00399970546 \ldots$ & \\
\hline 7 & 1.0 & 1.0 & & $0.05645188081 \ldots$ & \\
\hline 8 & 1.0 & 1.0 & & $0.00412064081 \ldots$ & \\
\hline 9 & 1.0 & 1.0 & & $0.00382840377 \ldots$ & \\
\hline 10 & 1.0 & 1.0 & & $0.00390041122 \ldots$ & \\
\hline 11 & 1.0 & 0.0 & + & $0.00326397468 \ldots$ & \\
\hline 12 & 1.0 & 1.0 & & $0.00529636720 \ldots$ & \\
\hline 13 & 1.0 & 1.0 & & $0.00495630790 \ldots$ & \\
\hline 14 & 1.0 & 1.0 & & $0.00345359330 \ldots$ & \\
\hline 15 & 1.0 & 1.0 & & $0.00419166415 \ldots$ & \\
\hline 16 & 1.0 & 1.0 & & $0.05341574664 \ldots$ & \\
\hline 17 & 1.0 & 1.0 & & $0.05022928811 \ldots$ & \\
\hline 18 & 0.0 & 1.0 & & $0.00395600124 \ldots$ & \\
\hline 19 & 1.0 & 1.0 & & $0.01035312046 \ldots$ & \\
\hline 20 & 1.0 & 1.0 & & $0.01046901704 \ldots$ & \\
\hline 21 & 1.0 & 1.0 & & $0.00456434121 \ldots$ & \\
\hline 22 & 1.0 & 1.0 & & $0.00373915666 \ldots$ & \\
\hline 23 & 1.0 & 1.0 & & $0.00343418029 \ldots$ & \\
\hline 24 & 1.0 & 1.0 & & $0.00620874308 \ldots$ & \\
\hline 25 & 1.0 & 1.0 & & $0.01354305304 \ldots$ & \\
\hline 26 & 1.0 & 1.0 & & $0.03166041464 \ldots$ & \\
\hline 27 & 1.0 & 1.0 & & $0.01073837378 \ldots$ & \\
\hline 28 & 1.0 & 1.0 & & $0.00745656459 \ldots$ & \\
\hline 29 & 1.0 & 1.0 & & $0.00358887639 \ldots$ & \\
\hline 30 & 1.0 & 0.0 & & $0.00334732554 \ldots$ & \\
\hline 31 & 1.0 & 1.0 & & $0.00553914760 \ldots$ & \\
\hline 32 & 1.0 & 1.0 & & $0.00342919983 \ldots$ & \\
\hline 33 & 1.0 & 1.0 & & $0.00408324426 \ldots$ & \\
\hline 34 & 1.0 & 0.0 & & $0.00330202388 \ldots$ & \\
\hline 35 & 1.0 & 1.0 & & $0.00421156660 \ldots$ & \\
\hline 36 & 1.0 & 1.0 & & $0.02011947619 \ldots$ & \\
\hline 37 & 1.0 & 1.0 & & $0.04797905933 \ldots$ & \\
\hline 38 & 1.0 & 1.0 & & $0.05102679762 \ldots$ & \\
\hline 39 & 1.0 & 1.0 & & $0.02864203757 \ldots$ & \\
\hline 40 & 1.0 & 1.0 & & $0.02781655886 \ldots$ & $L$ \\
\hline 41 & 1.0 & 1.0 & & $0.00353047890 \ldots$ & $\nabla$ \\
\hline
\end{tabular}

\section{CONCLUSION}

A new approach to the diagnosis of liver disease using Neutrosophic-based Decision Support approach was developed in this research and its performance was measured. In this research work the proposed use of the Neutrosophicbased Decision Support System takes in a set of signs and symptoms as well as laboratory results which is analyzed and the output is a confusability measurement which is then used to conclude whether that instance is a liver patient or not. The crux of this work is to compare the performance of our proposed system with the traditional multiyear neural network as well as assay the suitability of our proposed system for the diagnosis of liver disease. It was observed that our approach performed better with $96.41 \%$ accuracy using a confusability measurement threshold of 0.003278 hence suitable for the diagnosis of liver diseases. This would ultimately lead to high quality diagnosis and service delivery in medical institutions.

\section{REFERENCES}

[1] Adeniji K. A and Anjorin A.S. (2002). Histopathological Assessment of the pattern of liver cirrhosis in a tropical population. Afr J Med Sci 2002; 31: 367-9

[2] Ansari A. Q, Biswas, R. and Aggarwal S. (2013) . Neutrosophic classifier: An extension of fuzzy classifier. Applied Soft Computing, 13(1) 563-573.
[3] Ashwani, K and Neelam S.(2017). Categorization of Liver Disease Using Classification Techniques, International Journal for Research in Applied Science \& Engineering Technology (IJRASET) 826

[4] Dhamodharan S. (2014). Liver Diseases Prediction Using Bayesian Classification. National Conference on Advanced Computing, Applications \& Technologies, ISSN: 2320-0790, 1-3, 2014.

[5] Drăgulescu, D and Albu, A(2007). Medical Predictions System volume 4(3).

[6] Gulia, V.R. and Rani P. (2014). Liver Patient Classification Using Intelligent Techniques, International Journal of Computer Science and Information Technologies, 5 (4) , 5110-5115

[7] Jankisharan, P, Rajan, V, Jagdish, M and Sanjay, P. (2014). Liver Patient Classification Using Intelligence Techniques. International journal of advanced research in computer science and software enginerring.4 (2).

[8] Javad. S and Sartakht, J. S. (2011). Hepatitis disease diagnosis using a novel hybrid method, Elsevier, 570-579.

[9] Kavitha B., Karthikeyan S. and Sheeba M. P. (2011): An ensemble design of intrusion detection system for handling uncertainty using Neutrosophic Logic Classifier. 28 (2012) 88-96

[10] Khorashadizade N and Rezaei, H. (2015): A New method for rapid diagnosis of Hepatitis disease based on reduction feature and machine learning-Journal of Advanced Computer Science and Technology . 4(1) 148-155

[11] Mohammed, A. Alghambi, S. G. and Afshar M. A.(2015). Physician's decision process for disease diagnosis of overlapping syndrome in liver disease using soft computing. International Journal of Soft Computing and Engineering.(IJSCE).4(6)28-33. 
[12] Nwokediuko S.C, Osuala P.C, Uduma U.V, Alaneme A.K, Onwuka C.C, and Mesigo C. (2013). Pattern of Liver disease in Nigeria Tertiary Hospital. Niger J Clin Pract. 16,339-42

[13] Obeidat, I., Al-khasawneh, A, Bsoul M. (2013). Computer based clinical decision support system for hepatitis disease diagnosisInternational journal of advancements in computing technology. 5; 15-22.

[14] Okpako. A.E and Asagba P.O (2017). A Framework for Diagnosing Confusable Diseases using Neutrosophic-based Neural Network. Neutrosophic Sets and Systems. 16, 28-34.

[15] Pooja. S and Yukti, K. (2016). An Efficient Classifier using Multilayer Perceptron for Classification of Liver patient.
International Journal for Research in Applied Science \& Engineering Technology (IJRASET). 4(6); 646-648.

[16] Ramana. B. V. and Surendra, P.B. (2012). Liver Classification Using Modified Rotation Forest. International Journal of Engineering Research and Development, 1( 6) 17-24, 2012.

[17] Tahseen A. Jilani, H. Y and Madiha M. Y.(2011). PCA-ANN for classification of Hepatitis $\mathrm{C}$ patients- International journal of computer applications

[18] Vijayarani S. and Dhayanand S. (2015). Liver Disease Prediction using SVM and Naïve Bayes Algorithms. International Journal of Science, Engineering and Technology Research (IJSETR) 4(4).

[19] Zadeh L.A. (1996). Fuzzy logic = Computing with Words. IEEE Trans. on Fuzzy Systems, 4, 103-111. 